\title{
Plant Monitoring System for Greenhouse Management
}

\author{
B. Kiran Bala, R. Arjun, S. Gokul, V. Karthik, N. Mathes
}

\begin{abstract}
This system is intended to monitor the extinct plants. Generally, these kinds of system will be deployed in the garden of our home, but we made them suitable for the greenhouse in monitoring the individual plant. This system has light, temperature, humidity and moisture sensors are available, our work to monitor the plant will be much easier. This entire thing will be ease by the concept of Cloud and IoT. We need a microcontroller that connects the cloud via the internet. Due to this purpose, we discovered the Bolt IoT module integrated with its cloud. Since this module is having only a single analog pin to read the data, we interfaced with the Arduino UNO with the Bolt by UART protocol. By connecting the required sensors with the analog pins of the Arduino board, we collected the data and pushed them to the cloud to represent the required data in a graphical form.
\end{abstract}

Keywords: Temperature, Humidity, Moisture, Arduino, Bolt.

\section{INTRODUCTION}

We used the various sensors for this system such as Light Intensity sensor, Temperature sensor, Humidity Sensor, Moisture Sensor for readings. Bolt is an IoT support module that is powered by ESP8266. It supports $2.4 \mathrm{GHz}$ Wi-Fi band and Arduino Uno is powered by the ATmega328P processor. We chosen these boards since both of them have the feature for the serial data communication. This made possible by implementing the UART(Universal Asynchronous Receiver/Transmitter) mechanism. The interfacing between both of the boards was done by using the hardware serial interfacing technique. This interfacing requires the appropriate interfacing code uploaded to the Arduino Uno. As we mentioned above, The sensors were get connected and the data collection is done in the following manner. We connected the Light sensor to the A0 pin of Arduino Uno which we used were LDR(Light Dependent Resistor), It

Revised Manuscript Received on February 05, 2020.

* Correspondence Author

B. Kiran Bala*, Computer Science and Engineering, K.Ramakrishnan College of Engineering, Trichy, Tamil Nadu, India.

E-mail: kiranit2010@gmail.com

R. Arjun, Computer Science and Engineering, K.Ramakrishnan College of Engineering, Trichy, Tamil Nadu, India.

S.Gokul, Computer Science and Engineering, K.Ramakrishnan College of En gineering, Trichy, Tamil Nadu, India.

V. Karthik, Computer Science and Engineering, K.Ramakrishnan College of Engineering, Trichy, Tamil Nadu, India.

N. Mathes, Computer Science and Engineering, K.Ramakrishnan College of Engineering, Trichy, Tamil Nadu, India.

(C) The Authors. Published by Blue Eyes Intelligence Engineering and Sciences Publication (BEIESP). This is an open access article under the CC BY-NC-ND license (http://creativecommons.org/licenses/by-nc-nd/4.0/) measures the light intensity level which helps to maintain the light lumen level. Since it is the first analog pin that gets connected, It is data was read by the Bolt firstly by the UART transfer mechanism [1].

Then we connected the Temperature sensor to the A1 pin of Arduino Uno which we used were LM35, It helps to keep us maintain the exact temperature levels for the specified plants. This particular sensor is having a withstand capacity of about $-55^{\circ} \mathrm{C}$ to $150{ }^{\circ} \mathrm{C}$. Under this threshold temperature, it works fine. Since it is connected to the A1 pin, It's data was read by Bolt secondly [2].

Later we connected the moisture sensor to the A2 pin of Arduino Uno. Here we used the Capacitive based probe sensor to measure the values. We used it to overcome the drawback of the resistive sensor such as corrosion, inaccuracy. This help to maintain the moisture level in the soil accurately since we connected to the A2 pin, It is data was sent as the third one to the Bolt module and block diagram shown in the Fig 1 [3].

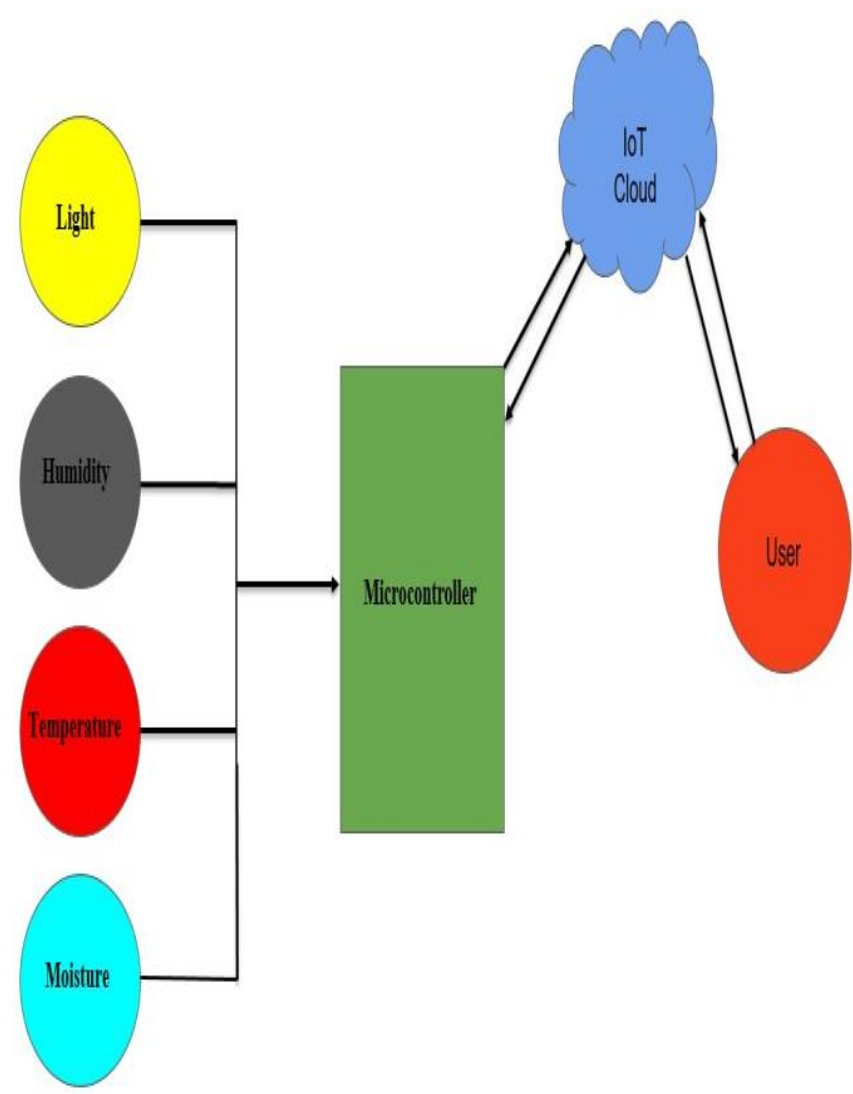

Fig. 1. Basic Block Diagram of the system 


\section{Plant Monitoring System for Greenhouse Management}

\section{MATERIALS AND METHODS}

Regarding the Humidity sensor, we connected them to the A3 pin of the Arduino Uno. Here we used the DHT11 sensor to measure the humidity level in the air. It also includes the inbuilt temperature sensor in the surroundings. But we used the dedicated sensor for temperature monitoring. Since it is connected to the A3 pin, The data read were fourthly by the Bolt module from the Arduino [4].

Each of the sensors we used was decided in a manner that adapts the low power management. The following are the operating voltage of each sensor. Here we power up the Bolt by $5 \mathrm{v}$. Then the Arduino Uno was interfaced with the Bolt by connecting the $5 \mathrm{v}$ and GND pins from the Bolt to the Arduino Uno. Since we have chosen the UART protocol for communicating the data to the Bolt module from the Arduino. We connected the Tx pin of Arduino with the Rx pin of the Bolt module. Then, vice versa we connected the Rx pin of the Uno board to the Tx pin of the Bolt module. These connections were done to make feasible the communication between both of the boards. Due to this, the data read will be possible from the Uno board [5].

In the Bolt Cloud after receiving the data, we configured it to simulate the data as a line graph. After the representation, additionally, we introduced the alert system to intimate users when it reaches a certain threshold for a particular aspect. The alert system can alert us by sending us the push notifications via dedicated bolt platform application as well as SMS to the individuals and Figure 2 \& Figure 3 shows the responsibilities of the process [6].

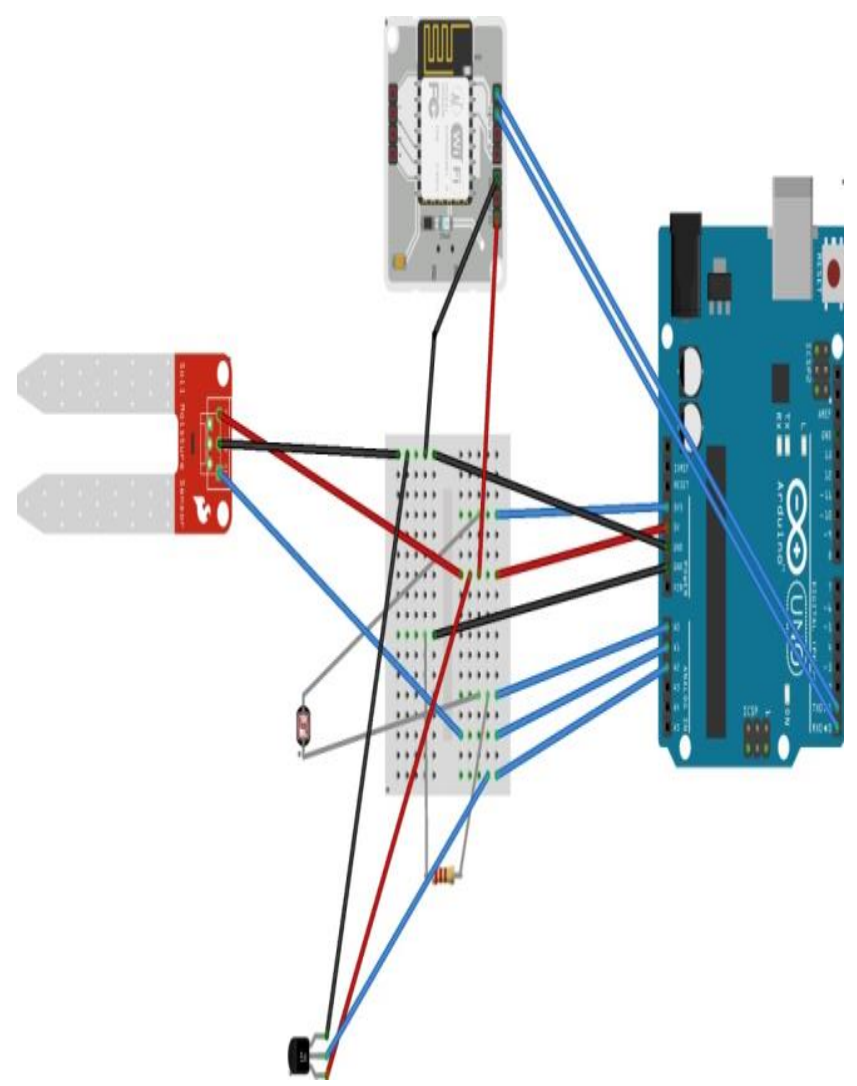

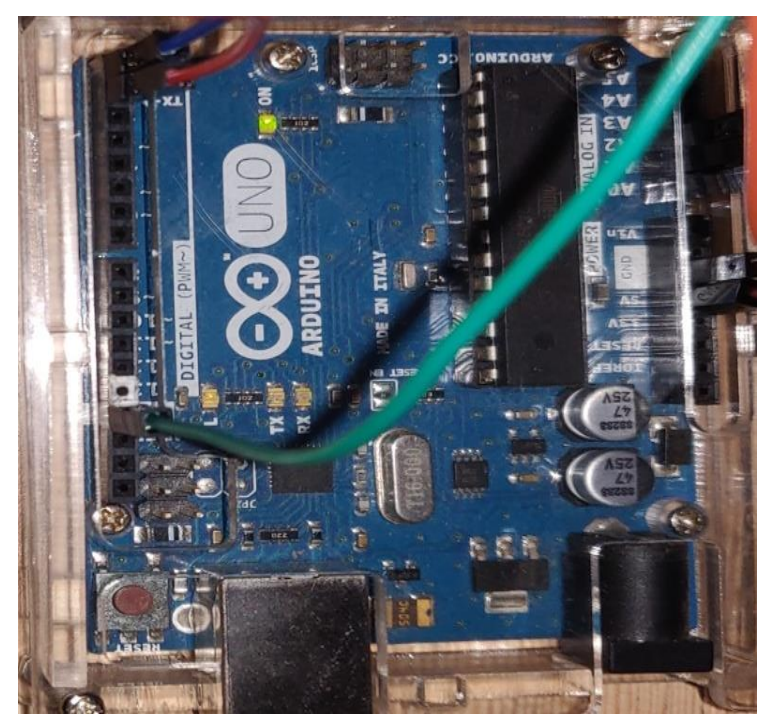

Fig. 3. Physical set of the system

This system helps us to keep track of our plants indigenously. This makes us monitor the plants at extinct in a hassle-free manner. Due to the application of the cloud manipulation and IoT, retrieval of the data and manipulating it for representing the collected data will become easier for us to keep track of it. These entire things were done real-time and updation will be done instantly due to the IoT cloud implementation. So, It helps monitor the plant in a real-time manner.

\section{IMPLEMENTATION}

This system mainly used to concentrate the light, temperature, humidity and moisture if any variation occur this system will alert the user through graph as well as SMS and in the Figure 4 \& Figure 5 shows the variation in the graph according to the variation of graph.

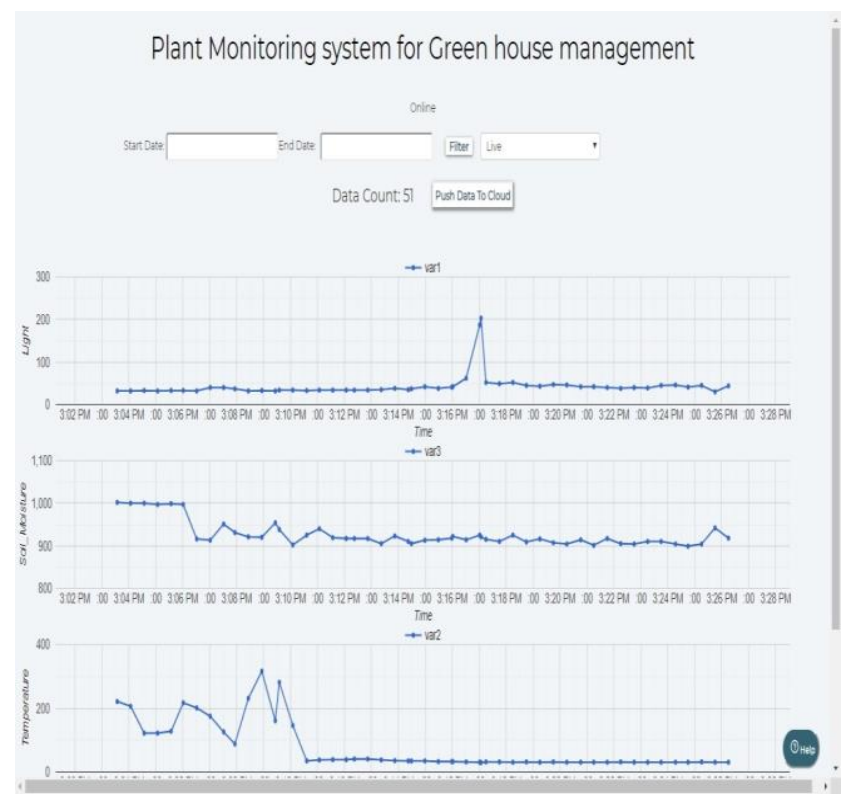

Fig. 4. Normal Graph

Fig. 2. Connection Diagram of the system 


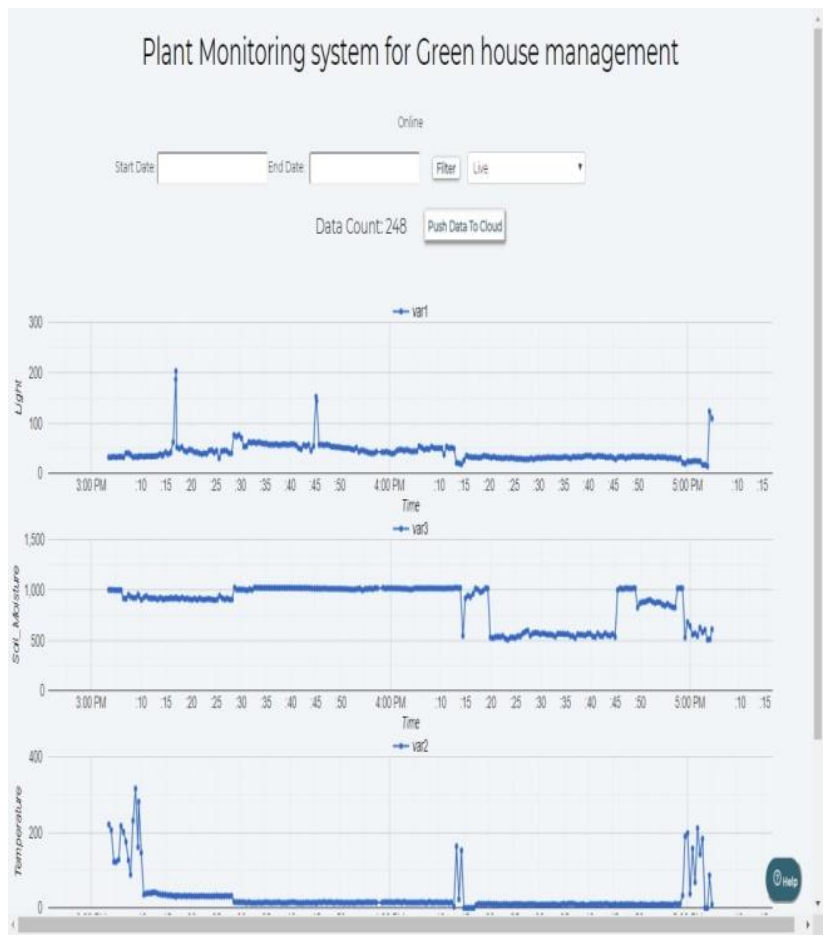

Fig. 5. Variation in Graph

\section{CONCLUSION}

This system helps the e-agriculture system in future from the implementation as well in material and methods clearly explained about the sensors and work flow of the system even in implementation part also the variation among the graph is also shown. So, this is suitable for the agriculture sector leads to one green revolution in upcoming years.

\section{REFERENCES}

1. Kiran Bala B, Balakumar A, 'The Combination of Steganography and Cryptography for Medical Image Applications', Biomedical and Pharmacology Journal, Volume 10, Issue 4, Year 2017

2. Kiran Bala B, Lourdu Joanna J, 'Multi Modal Biometrics Using Cryptographic Algorithm', European Journal of Academic essays, ISSN: 2183 1904, vol 1 Issue 1, February 2014.

3. Kiran Bala B, Audithan S, 'Wavelet And Curvelet Analysis For The Classification Of Microcalcifiaction Using Mammogram Images' Publisher: IEEE, ISBN: 978-1-4799-7986-8, Page: 517-521.

4. Kiran Bala B, 'A Novel Approach To Identify The Micro Calcification Images', Journal of Chemical and Pharmaceutical Sciences , JCHPS Special Issue 2: February 2017, Page 190-192.

5. Kiran Bala B, Infant Raj I, „Comparative and identification of exact frequency domain approaches by using mammogram images ${ }^{\text {ee }}, 2017$ IEEE International Conference on Power, Control, Signals and Instrumentation Engineering (ICPCSI), Publisher: IEEE, Year 2018.

6. Kiran Bala B, 'A Novel Approach To Generate A Key For Cryptographic Algorithm', Journal of Chemical and Pharmaceutical Sciences , JCHPS Special Issue 2: February 2017, Page 229-231.

\section{AUTHORS PROFILE}

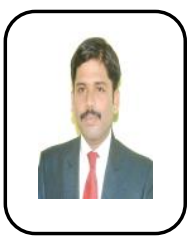

B. Kiran Bala working as an Assistant Professor in K.Ramakrishnan College of Engineering, Trichy, Tamil Nadu, India and published more than 50 papers in Image processing.

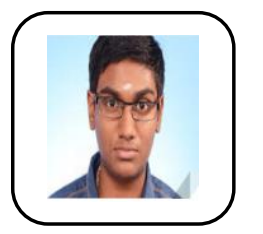

R. Arjun, Studying in IV year, Department of Computer science and engineering, K.Ramakrishnan College of Engineering, Trichy, Tamil Nadu, India

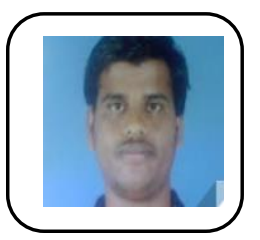

S. Gokul, Studying in IV year, Department of Computer science and engineering, K.Ramakrishnan College of Engineering, Trichy, Tamil Nadu, India

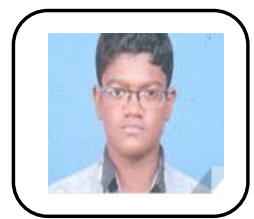

V. Karthik, Studying in IV year, Department of Computer science and engineering, K.Ramakrishnan College of Engineering, Trichy, Tamil Nadu, India

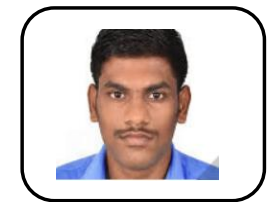

N. Mathes, Studying in IV year, Department of Computer science and engineering, K.Ramakrishnan College of Engineering, Trichy, Tamil Nadu, India 